\title{
Pratiques enseignantes pour l'apprentissage de l'écriture en grande section de maternelle et au cours préparatoire en France
}

Teaching handwriting in late kindergarden and first year of elementary French schools

Marie Baraud, Blandine Bril et Nadja Acioly-Régnier

\section{OpenEdition Journals}

Édition électronique

URL : https://journals.openedition.org/educationdidactique/3312

DOI : 10.4000/educationdidactique.3312

ISSN : 2111-4838

Éditeur

Presses universitaires de Rennes

\section{Édition imprimée}

Date de publication : 21 décembre 2018

Pagination : 73-88

ISBN : 978-2-7535-7711-4

ISSN : 1956-3485

Référence électronique

Marie Baraud, Blandine Bril et Nadja Acioly-Régnier, « Pratiques enseignantes pour l'apprentissage de l'écriture en grande section de maternelle et au cours préparatoire en France », Éducation et didactique [En ligne], 12-2 | 2018, mis en ligne le 21 décembre 2020, consulté le 23 août 2022. URL : http:// journals.openedition.org/educationdidactique/3312 ; DOI : https://doi.org/10.4000/ educationdidactique.3312 


\title{
PRATIQUES ENSEIGNANTES POUR L'APPRENTISSAGE DE L'ÉCRITURE EN GRANDE SECTION DE MATERNELLE ET AU COURS PRÉPARATOIRE EN FRANCE
}

\author{
Marie Baraud, groupe de recherche Apprentissage et contexte, \\ École des hautes études en sciences sociales \\ Blandine Bril, groupe de recherche Apprentissage et contexte, \\ École des hautes études en sciences sociales; \\ laboratoire Éducation, discours et apprentissage (EDA), université Paris-Descartes \\ Nadja Acioly-Régnier, ESPE ; université Claude Bernard Lyon1, \\ UMR 5191 ICAR (Interactions, corpus, apprentissage, représentations)
}

Les programmes de l'Éducation nationale en France prévoient une liberté pédagogique, ce qui laisse les enseignants libres du choix de leurs méthodes. Nous avons cherché à travers cette enquête conduite dans deux académies (Grenoble et Lyon), à faire un état des lieux des méthodes d'enseignement de l'écriture cursive en GS et en CP à partir d'un questionnaire sur la manière dont les enseignants perçoivent les difficultés liées au geste graphomoteur de l'écriture cursive, et adaptent leurs stratégies d'enseignement en conséquence. Lanalyse des questionnaires montre une grande diversité de pratiques tant en nombre de séances centrées explicitement sur l'acquisition du graphisme proprement dit que sur les méthodes et stratégies utilisées. Cependant, malgré cette grande variété de méthodes, l'enseignement est pour la grande majorité des enseignants centrée sur l'acquisition de la dynamique du geste et non sur la forme de la lettre. On peut conclure que la liberté laissée au corps enseignant par des programmes souvent présentés comme manquant de clarté les conduit en quelque sorte à un certain «bricolage " construit à partir des diverses ressources disponibles.

Mots-clés : geste graphique, écriture cursive, enquête, enseignement, grande section maternelle, cours préparatoire.

Teaching handwriting in late kindergarden and first year of elementary French schools

Official french curricula grant an educational freedom to teachers providing full flexibility of choosing teaching methods. The present survey conducted in two areas (Grenoble and Lyon) aimed at assessing the teaching of cursive-writing in late kindergarten and first year of elementary school in France. It was based on a questionnaire surveying the difficulties teachers perceive regarding the acquisition of the cursive writing grapho-motor movement, and how they adapt their teaching strategies. The analysis of the questionnaires has shown a great diversity of practices. These practices are related to the number of lessons explicitly working on the acquisition penmanship itself as well as the strategies and methods. However, despite this variety of methods, the training prioritizes the movement dynamic rather than on the form of the letter. The liberty granted to teachers by curricula - which are often said to be lacking clarity - lead them to a process of "bricolage" using the variety of resources available to organize their teaching.

Keywords: graphic movement, cursive writing, questionnaire, teaching, kindergarten, first year of primary school.

Nous remercions chaleureusement les enseignants anonymes qui ont pris le temps de répondre à ce questionnaire. Cette étude a été financée par l'ANR GESTEC (ANR-13-APPR-0005) « Le geste technique et son apprentissage », dont Blandine Bril est responsable scientifique. 


\section{INTRODUCTION}

L'instauration en 1882 de l'école obligatoire a permis de généraliser en France l'accès à l'écriture manuscrite ${ }^{1}$. Depuis lors, l'écriture est enseignée aux enfants à partir de 5 ou 6 ans et l'organisation de cet enseignement est définie par tout un ensemble de textes officiels : les lois, les décrets d'application ainsi que les arrêtés qui fixent les programmes, ces textes définissant l'enseignement en termes d'objectifs à atteindre pour chaque cycle (Baraud, Bril, Hoibian, Bizaguet, Acioly-Régnier, 2016).

Dans le but d'évaluer la manière dont les enseignants déclarent construire l'environnement dans lequel se déroule cet apprentissage, une enquête par internet sous forme de questionnaire a été proposée à l'ensemble des classes de grande section (GS) et de cours préparatoire (CP) des écoles maternelles et primaires de deux académies de la région AuvergneRhône-Alpes. L'objectif était de tenter d'évaluer la manière dont les pratiques enseignantes assistent l'appropriation par l'enfant du geste d'écriture.

En effet, du fait du principe de liberté pédagogique, le choix des méthodes est laissé aux enseignants bien que des documents d'accompagnement soient édités par le ministère de l'Éducation nationale et que tout un ensemble d'ouvrages, souvent écrits par des enseignants, offrent de nombreuses suggestions de méthodes afin de mettre en œuvre les programmes (Baraud et al., 2016). Cependant, une fois le cadre institutionnel tracé, comment cet apprentissage est-il organisé concrètement, dans chaque école et dans chaque classe ? Comment chaque professeur des écoles traduit-il/elle ces recommandations en activité pour chaque élève?

L'enquête que nous rapportons ici est basée sur les réponses de 226 enseignants de grande section de maternelle (GS) et de cours préparatoire (CP). Un ensemble de questions, dont un certain nombre étaient présentées sous forme de questions ouvertes, a permis d'évaluer divers aspects de l'activité d'enseignement tels que le temps dédié à cet enseignement, les ressources matérielles relatives à l'écriture dont disposent les classes, les stratégies d'enseignement, ou encore l'évaluation des difficultés des élèves dans le tracé des lettres et des mots. D'autres questions avaient pour but d'explorer la manière dont les enseignants interprètent dans leur pratique enseignante les recommandations des instructions officielles concernant l'apprentissage de l'écriture en GS et CP, de même que leur recours à des ressources autres tels que les manuels ou les livres du maitre.

C'est donc à l'analyse des stratégies déclarées par les enseignants ainsi qu'aux outils utilisés pour appuyer ces stratégies que nous nous intéresserons ici. Un travail préalable sur les textes de loi, les programmes et les supports d'enseignement a en effet permis de montrer un certain flou des directives destinées aux enseignants mais aussi une grande variété des supports disponibles (Baraud et al., 2016 ; Bril et al., 2016). L'objectif du présent article est ainsi double, d'une part évaluer la manière dont les enseignants organisent l'activité des élèves tout en mettant à profit la liberté pédagogique qui leur est offerte, et d'autre part analyser les stratégies pédagogiques en regard des propriétés inhérentes au geste d'écriture que nous envisageons ici en termes de contraintes fonctionnelles - dynamique de la trajectoire (sens et direction) et pression de l'outil scripteur sur le support.

L'approche adoptée privilégie donc deux aspects spécifiques et complémentaires de l'apprentissage de l'écriture manuscrite cursive : l'acquisition de la composante graphique de l'écriture et du geste et corrélativement les conditions d'exercice qui peuvent en faciliter l'apprentissage. En effet la manière dont l'enseignant aménage l'environnement de l'enfant et régule son activité va assister et contribuer à l'apprentissage du geste par le jeune scripteur.

\section{L'APPRENTISSAGE DE L'ÉCRITURE}

Avant de s'interroger sur les pratiques des enseignants, et donc sur les conditions de l'acquisition de l'écriture cursive, arrêtons-nous quelques instants sur l'objet de l'apprentissage : que doit apprendre l'enfant pour être capable de tracer des lettres aisément et de manière lisible? En d'autres termes en quoi consiste, d'un point de vue graphique, le fait d'être capable de laisser une trace correspondant à une lettre ou une succession de lettres et qui de surcroit ait un sens?

\section{L'objet de l'apprentissage : le geste graphique dans l'écriture cursive}

L'écriture alphabétique cursive implique une succession de déplacements de l'outil scripteur formant des lignes plus ou moins courbes. Il s'agit 
donc pour l'apprenant d'acquérir une stratégie permettant de transformer un mouvement se déroulant dans le temps en une trace ayant des propriétés spatiales particulières liées à la forme, la taille, l'inclinaison, l'alignement ou encore l'organisation spatiale des tracés (Bara et Gentaz, 2010 ; Lurçat, 1983). Ainsi l'une des exigences fonctionnelles ${ }^{2}$ de l'écriture consiste en des relations dynamiques entre la pointe de l'élément scripteur (stylo, crayon) et la surface du support (papier, ardoise, tableau, etc.). Il est courant de présenter l'écriture selon deux dimensions, l'une statique, la forme, et l'autre dynamique, la trajectoire (Vinter et Chartrel, 2010). Analysant la formation du tracé, Lurçat (1983) distingue la rotation et la translation, la rotation correspondant au sens du tracé, horaire ou antihoraire, et la translation correspondant à la direction générale du tracé, de gauche à droite pour de nombreux systèmes d'écriture en particulier pour les écritures latines et grecques, ou de droite à gauche, sens des écritures arabe ou hébraique par exemple. Le geste graphique serait ainsi caractérisé par la combinaison de ces deux mouvements. La maitrise de la composante graphique de l'écriture nécessite donc le contrôle de la trajectoire qui conditionne la forme de la lettre d'une part, et de la dynamique produisant la trajectoire d'autre part, auxquelles il faut ajouter une composante de pression qui crée les conditions de déplacement de la pointe de l'outil scripteur. Le mouvement est en effet contraint par la nature du frottement entre les deux surfaces ou plus exactement par les forces de frottement en jeu.

Pour Lurçat (1983), l'acquisition de la forme précède celle du sens du mouvement, ce qui a pour conséquence la nécessité d'une pédagogie de l'écriture tournée vers la trajectoire plutôt que vers le résultat, c'est à dire la forme.

De nombreux travaux ont analysé les caractéristiques de l'écriture selon l'âge et/ou le niveau scolaire (voir par exemple : Chartrel et Vinter, 2004 ; BosgaStork, Bosga, Meulenbroek, 2011, Karlsdottir, 1996 ; Meulenbroek et Van Galen, 1988) et montré l'évolution de la qualité de la production des formes écrites, de la maitrise du geste graphomoteur dans le sens d'une meilleure fluidité de mouvement allant de pair avec une vitesse d'écriture plus rapide et mieux maitrisée, avec une diminution du nombre de pics d'accélération; dans le même temps, la longueur des trajectoires diminue pour se stabiliser vers 6 ou 7 ans alors que la pression augmente (Vinter et Chartrel,
2008). D'autres études ont cherché à déterminer le rôle des ressources sensori-motrices, qu'elles soient perceptives, cognitives, attentionnelles, linguistiques (Bara et Gentaz, 2010) ainsi que les principes de l'intégration visuo-motrice (Maldarelli et al., 2015 ; Vinter et Chartrel, 2010) ou tactile/kinesthésique (Bara et Gentaz, 2011 ; Chartrel et Vinter, 2008 ; Watkins et Nobles, 2011).

Les travaux expérimentaux plus particulièrement pertinents ici sont ceux détaillant l'effet différentiel de l'entrainement selon le type d'informations fourni à l'apprenant, qu'elles soient verbales - description verbales des formes à tracer, ou sensorielles - visuelles, haptiques, kinesthésique. Si la forme des lettres relève d'informations visuelles, l'association d'instructions verbales sur le tracé de même qu'une mise en évidence de la dynamique de sa trajectoire augmente la portée de l'entrainement. L'efficacité d'un enseignement explicite donnant des informations sur la forme du tracé et direction du mouvement associé à sa réalisation s'avère plus efficace que l'explication seule ou la démonstration seule (Kaiser et al. 2011). En outre une explicitation de la production réalisée par l'enfant lui-même est un facteur renforçateur.

Vinter et Chartrel (2010) ont démontré l'effet bénéfique d'un entrainement visuel dynamique (le modèle de lettre développe la dynamique de la trajectoire) comparé à un modèle statique (pas d'information sur la direction du mouvement), associé à la fréquence de l'activité motrice de l'enfant (nombre plus ou moins important de productions du mouvement de tracé). Leurs résultats suggèrent en outre qu'une information visuelle dynamique pourrait influencer le contrôle de la taille du tracé à l'instar de contraintes spatiales (taille des réglures).

Par ailleurs, un entrainement multisensoriel visuo-haptique s'avère plus efficace qu'un entrainement basé uniquement sur les informations visuelles (Gimbert, Gentaz et Mazens, 2013 ; Bara et Gentaz, 2010).

Ainsi ces différentes études mettent clairement en évidence l'effet différentiel de modalités variées de l'entrainement sur la qualité de l'écriture. Ainsi l'objectif du présent travail consistait à évaluer dans quelle mesure les stratégies pédagogiques rapportées par les enseignants exploitent les entrainements sensori-moteurs mis en évidence dans les travaux expérimentaux. 
Nous plaçant dans la lignée des travaux de Bernstein (1967, 1996 ; Newell, 1989), nous considérons que l'apprentissage est basé sur un processus d'exploration et d'exploitation des différentes contraintes de la situation qui doivent conduire l'apprenant à satisfaire les contraintes de la tâche (Bril, 2015), ici les caractéristiques graphiques de l'écriture, principalement trajectoire et pression de l'outil scripteur. C'est dans cette optique que les pratiques des enseignants seront analysées selon qu'elles permettent à l'enfant (l'apprenant) de découvrir et de maitriser ces différentes dimensions fonctionnelles de l'écriture en sollicitant ses ressources sensori-motrices.

\section{L'organisation du contexte de l'apprentissage}

Ainsi la découverte des propriétés de l'action et la capacité à les satisfaire de manière optimale n'est pas simplement une affaire individuelle. Elle s'appuie sur l'organisation du « contexte » de l'apprentissage qui crée pour l'apprenant un « espace d'actions encouragées » (Reed, 1993 ; Reed et Bril, 1996). Cet espace d'actions encouragées, peut être décrit selon deux dimensions : l'une spécifie l'organisation matérielle de l'environnement de l'apprenant, et donc la mise à disposition d'objets, d'opportunités d'actions, etc. ; l'autre dimension est relative à l'activité pédagogique du tuteur et passe nécessairement par la diversité des expériences organisées de manière plus ou moins directe par les spécificités des éléments de l'environnement de l'apprenant. Cette médiation de l'environnement a été souvent minimisée dans les études sur l'apprentissage, alors qu'elle est centrale dans l'explication de la construction du contrôle de l'action. Dans le cas qui nous intéresse, l'apprentissage de la composante graphique de l'écriture cursive, cet environnement et sa dynamique sont donnés par l'organisation des situations qui de près ou de loin amènent l'enfant à se familiariser et à s'entrainer à cette activité, que ce soit dans le cadre scolaire, familial ou dans tout autre contexte social. C'est ici au contexte scolaire que nous nous intéressons, contexte dans lequel le maitre est investi de ce rôle essentiel de médiateur, d'organisateur de l'expérience de l'enfant, en d'autres termes de l'espace d'action de l'élève (Valsiner et Van der Veer, 1993 ; Reed, 1993 ; Reed et Bril 1996). Reprenant l'idée développée par J. Gibson de la nécessaire «éducation de l'attention »
(Gibson, 1966), nous nous intéressons tout particulièrement à la manière dont l'enseignant aménage l'expérience de l'enfant, et en particulier à l'organisation matérielle de l'environnement, et donc la mise à disposition d'objets, d'opportunités d'actions d'une part, et d'autre part à la manière dont l'enseignant sollicite l'attention de l'élève sur les caractéristiques fonctionnelles de l'écriture. L'espace d'actions « encouragées » ainsi créé doit permettre à l'élève un processus d'exploration, de découverte et d'intégration des propriétés de la tâche.

Si de nombreux travaux se sont intéressés à la manière dont l'enfant acquiert progressivement la capacité à écrire, peu de travaux se sont penchés sur l'organisation de cet apprentissage, c'est à dire sur l'expérience proprement dite nécessaire à l'apprentissage en contexte réel - dans le cas présent, en classe. Les quelques (rares) travaux s'intéressant à la structuration de l'enseignement de l'écriture sont plus particulièrement issus de préoccupations liées aux questions de remédiation pour les enfants ayant des problèmes d'apprentissage du graphisme (Asher, 2006 ; Graham, 2008), ou à la nécessité de l'apprentissage de l'écriture cursive comparativement à l'écriture script (Bara, Morin, Montésinos-Gelet \& Lavoie, 2011) ou même à l'écriture manuscrite proprement dite préalablement à l'écriture sur clavier (Longcamp et al., 2005). C'est tout particulièrement le cas d'une recherche réalisée aux États-Unis par Graham (2008) il y a une dizaine d'années. Partant du constat qu'une mauvaise maitrise de la composante graphique de l'écriture pouvait avoir un impact négatif sur l'expression écrite proprement dite du fait d'une charge cognitive orientée sur l'acte graphique au détriment du message exprimé, Graham (2008) a mené une enquête sur les stratégies d'enseignement de l'écriture manuscrite aux États-Unis. Ainsi pour cet auteur, l'enseignement de l'écriture revêt une importance particulière car il va indirectement impacter l'ensemble des apprentissages scolaires. Il devient alors essentiel d'avoir une bonne connaissance des pratiques des enseignants. L'objectif de son étude était donc en particulier d'évaluer le lien entre le temps consacré à cet apprentissage de manière explicite et implicite, le contenu de l'enseignement et les performances de l'élève. Cette étude nous servira de base de comparaison avec les résultats de l'enquête que nous avons menée en France.

On constate donc que si un nombre important de travaux s'est intéressé aux mécanismes psycho- 
moteurs sous-jacents à l'acquisition de l'écriture, peu se sont penchés sur la manière de l'enseigner. Ceci est d'autant plus surprenant que les liens entre la maitrise de l'écriture cursive et les autres apprentissages sont connus. La maitrise de l'écriture manuscrite facilite l'expression écrite, donne à l'élève plus de chances d'avoir de bons résultats scolaires et augmente l'estime de soi (Feder et Majnemer, 2007 ; Sheffield, 1996).

Dans la suite de cet article nous analyserons les pratiques des enseignants en essayant d'évaluer la manière dont les caractéristiques fonctionnelles du graphisme, à savoir les trois dimensions fonctionnelles de l'action d'écriture ou tracé rappelées ci-dessus (direction, pression et forme), sont intégrées dans l'enseignement. Après avoir décrit l'échantillon des enseignants ayant répondu au questionnaire, et avoir donné la manière dont ce questionnaire a été conçu, nous nous concentrerons sur trois grands axes de l'organisation de l'enseignement auxquels les enseignants ont fait référence. Il est important de considérer tout d'abord les ressources auxquelles les enseignants ont accès, qu'elles soient institutionnelles ou didactiques. Nous verrons ensuite les stratégies développées par les enseignants ainsi que les supports matériels utilisés. Cette section se conclura sur l'évaluation des difficultés que pose l'écriture cursive. Nous terminerons par un aperçu de la manière dont les enseignants envisagent l'introduction des outils numériques et leur possible entrée en conflit avec la nécessité d'apprendre l'écriture cursive.

\section{MÉTHODE}

Afin de savoir quelles sont les pratiques quotidiennes d'enseignement et d'apprentissage de l'écriture cursive dans les classes de grande section de maternelle et de cours préparatoire françaises, nous avons mené une enquête par questionnaire dans deux académies de la région Auvergne-Rhône-Alpes. Une version en ligne du questionnaire a été envoyée par e-mail aux 3495 écoles élémentaires publiques des académies de Grenoble et de Lyon fin septembre 2015. Nous avons obtenu 226 réponses, toutefois, ne disposant pas du nombre d'enseignants concernés, nous ne pouvons fournir le taux de réponse obtenu.

\section{Échantillon}

Le tableau ci-dessous présente les caractéristiques générales des participants à l'enquête. Les répondants étaient âgés de 24 à 65 ans, avec une moyenne de 42 ans, et avaient entre 1 et 42 années d'expérience, avec une moyenne de 17,1 ans. Tous étaient enseignants de grande section (GS), de cours préparatoire (CP) ou de double niveau GS-CP.

Notre échantillon montre une surreprésentation des zones rurales au détriment des zones urbaines et un nombre important de classes de double niveau GS de maternelle et cours préparatoire. Nous avons décidé de garder l'ensemble des réponses au questionnaire malgré ce biais, car ayant envoyé le questionnaire à toutes les écoles de l'académie, avec un

1. Caractéristiques des répondants

\begin{tabular}{|l|l|l|l|l|l|}
\hline Niveau & Genre & \multicolumn{1}{l|}{ Secteur } \\
\hline CP & $59 \%(\mathrm{~N}=133)$ & Masculin & $8 \%(\mathrm{~N}=19)$ & Rural & $64 \%(\mathrm{~N}=145)$ \\
\hline GS & $10 \%(\mathrm{~N}=22)$ & Féminin & $90 \%(\mathrm{~N}=203)$ & Urbain & $31 \%(\mathrm{~N}=69)$ \\
\hline GS, CP & $26 \%(\mathrm{~N}=58)$ & Non rép. & $2 \%(\mathrm{~N}=4)$ & Urb. et rural & $3 \%(\mathrm{~N}=7)$ \\
\hline Non rép. & $6 \%(\mathrm{~N}=13)$ & & & Non rép. & $2 \%(\mathrm{~N}=5)$ \\
\hline Tranche d'âge & & Expérience \\
\hline Inf. 30 & $7 \%(\mathrm{~N}=15)$ & Inf. 10 & $21 \%(\mathrm{~N}=48)$ \\
\hline $30-40$ & $32 \%(\mathrm{~N}=73)$ & $11-20$ & $42 \%(\mathrm{~N}=94)$ \\
\hline $40-50$ & $35 \%(\mathrm{~N}=78)$ & $21-30$ & $20 \%(\mathrm{~N}=46)$ \\
\hline Sup. 50 & $17 \%(\mathrm{~N}=39)$ & Sup. 30 & $9 \%(\mathrm{~N}=20)$ \\
\hline Non rép. & $9 \%(\mathrm{~N}=21)$ & Non rép. & $8 \%(\mathrm{~N}=18)$ \\
\hline Total
\end{tabular}


taux de retour relativement faible, nous avons conclu que les enseignants ayant répondu correspondaient probablement à un ensemble motivé par cet enseignement, ce qui devait permettre de donner un tableau assez approfondi de cette question.

\section{Questionnaire}

Le questionnaire vise à mettre en évidence les pratiques des enseignants de CP et GS concernant l'enseignement-apprentissage de l'écriture cursive à travers des questions à choix multiples de différents types ainsi que des questions ouvertes. Il a pour but d'identifier les ressources utilisées par les enseignants (textes officiels, manuels, articles voire sites internet) pour organiser l'enseignement et proposer des supports aux élèves (modèles, cahiers, ardoises, etc.), ainsi que les pratiques d'enseignement et d'évaluation mises en œuvre dans le cadre de la classe. Enfin, deux questions ouvertes demandent aux participants une réflexion sur l'enseignement de l'écriture cursive et les outils numériques. Sa durée de passation est de 30 minutes environ

Le questionnaire se compose de sept parties dont nous donnons les intitulés et décrivons rapidement le contenu (cf. questionnaire en annexe) :

- "Méthodes»: composé de 16 questions concernant les méthodes et outils employés par les enseignants parmi lesquelles huit questions à choix multiple portant sur l'enseignement (supports disponibles et préférés, outils scripteurs, fréquence et durée des séances, type et utilisation de modèles, critères d'évaluation);

- « Textes et programmes » avec quatre questions sur le rapport des enseignants au programmes ;

- « Manuels» avec quatre questions sur l'utilisation et les connaissances des enseignants quant aux manuels (maisons d'édition, auteurs) ;

- «Évaluation » qui portait sur les difficultés fréquemment rencontrées par les élèves et les solutions adoptées par les enseignants, composée de 8 questions dont deux questions à choix multiple, deux questions de classement des difficultés et quatre questions ouvertes sur les lettres, enchainements et mots difficiles ainsi que sur la remédiation;

- « Profession » avec 3 questions sur la formation des participants, leur représentation de leur profession et le travail en équipe ;
- « Ouverture » comportant 2 questions liées à l'actualité (outils numérique et abandon de la cursive en Finlande) ainsi qu'une invitation à compléter librement leurs réponses ;

- une fiche signalétique.

Les questions ont été élaborées à partir de la littérature scientifique d'une part et d'une analyse des ressources à destination des enseignants d'autre part, afin d'évaluer la manière dont ces derniers construisent leur pratique pédagogique et donc organisent l'" espace d'actions encouragées des élèves ». La combinaison de questions fermées à choix multiple et de questions ouvertes permettait d'une part d'obtenir des statistiques assez précises des outils et des choix pédagogiques et d'autre part donnait la possibilité aux enseignants d'expliciter et de justifier les choix des procédures qu'ils utilisent.

\section{RÉSULTATS}

Les résultats de l'enquête seront regroupés selon quatre grands thèmes, dont certains seront déclinés en sous-thèmes : les ressources utilisées par les enseignants, les pratiques et stratégies d'enseignement déclarées par ceux-ci, les stratégies et solutions mises en œuvre face aux difficultés rencontrées par certains élèves et enfin la place du numérique dans l'apprentissage de l'écriture. Pour de nombreuses questions, les résultats sont donnés en pourcentage du nombre total d'enseignants. Par ailleurs les questions ouvertes ont été analysées en établissant des catégories a posteriori et en comptant le nombre d'occurrences de chaque catégorie. À des fins d'illustration les réponses seront parfois données telles qu'elles ont été exprimées par les enseignants.

\section{Ressources à disposition des enseignants}

Textes officiels, ouvrages spécialisés ou blogs enseignants, il existe toute une panoplie de ressources ayant pour objectif de faciliter et conseiller l'organisation de l'enseignement de l'écriture. Parmi ceuxci quels sont ceux que privilégient les enseignants ? Les programmes et textes officiels sont incontournables, $69 \%$ des enseignants s'y réfèrent bien qu'ils soient $74 \%$ à les trouver peu clairs. Rappelons qu'à la date à laquelle l'enquête a été menée, les 
programmes de maternelle venaient d'entrer en vigueur et les programmes de l'école élémentaire n'étaient pas encore parus : ces résultats concernent donc les programmes de 2008 pour l'élémentaire et les programmes de 2008 et 2015, que les enseignants venaient de découvrir et n'avaient donc pas encore eu le temps d'appréhender, pour la maternelle. Afin d'organiser l'enseignement du point de vue pratique, $32 \%$ se réfèrent aux manuels et livres du maitre et moins de $1 \%$ utilisent des ouvrages pédagogiques. En complément, les enseignants déclarent avoir recours à d'autres ressources et notamment à leur expérience personnelle et pour les trois quarts d'entre eux à des sites internet. Les ressources en ligne sont diverses puisqu'il peut s'agir de blogs rédigés par des enseignants afin de publier des supports pédagogiques, des emplois du temps, des exercices mais aussi des conseils quant au choix des manuels et des récits d'expérience. Il peut aussi s'agir de sites officiels ou de sites personnels de rééducateurs proposant des méthodes de remédiation pour l'écriture. Alors que peu d'enseignants (11\%) semblent participer à des réunions de concertation de l'équipe pédagogique de leur école, la moitié dit travailler en équipe avec leurs collègues afin, d'après eux, de garantir une continuité des apprentissages en harmonisant leurs pratiques et en utilisant un vocabulaire commun. Il leur parait également nécessaire de se concerter afin de bénéficier des connaissances de chacun en vue d'affiner leurs méthodes et surtout d'identifier les difficultés des élèves. Lorsqu'il y a absence de concertation elle est attribuée principalement au manque de temps, à la petite taille de l'école ou tout simplement que cela n'est pas ressenti comme une priorité.

Bien que la moitié des enseignants disent collaborer, la quasi-totalité (97\%) a déclaré avoir toute liberté de choix du manuel avec lequel travailler. De plus, face à la diversité des méthodes disponibles, la plupart des enseignants a déclaré ne pas être attaché à une maison d'édition particulière, que ce soit pour le livre du maitre ( $84 \%$ ) ou pour les cahiers et manuels destinés aux élèves (87\%). Cela peut signifier soit qu'ils combinent plusieurs méthodes, soit qu'ils changent de méthode au cours des années.

\section{Pratiques et stratégies d'enseignement}

Depuis 1977, les programmes ne donnent plus d'indication précise concernant la durée et la répartition hebdomadaire des séances d'écriture (Bril et al., 2016), la répartition des séances d'écriture dans l'emploi du temps de la classe est donc librement organisée par l'enseignant. Ainsi en moyenne une séance dure 21 minutes avec un écart se situant dans une fourchette allant de cinq minutes à une heure, ce qui donne une durée hebdomadaire d'environ $85 \mathrm{mn}$, avec là encore de très fortes variations allant de $10 \mathrm{mn}$ à cinq heures réparties en une à cinq séances par semaine. Toutefois, la moitié des enseignants

Figure 1. Répartition du nombre de séances dédiées à l'écriture par semaine (A), ainsi que les durées des séances (B)

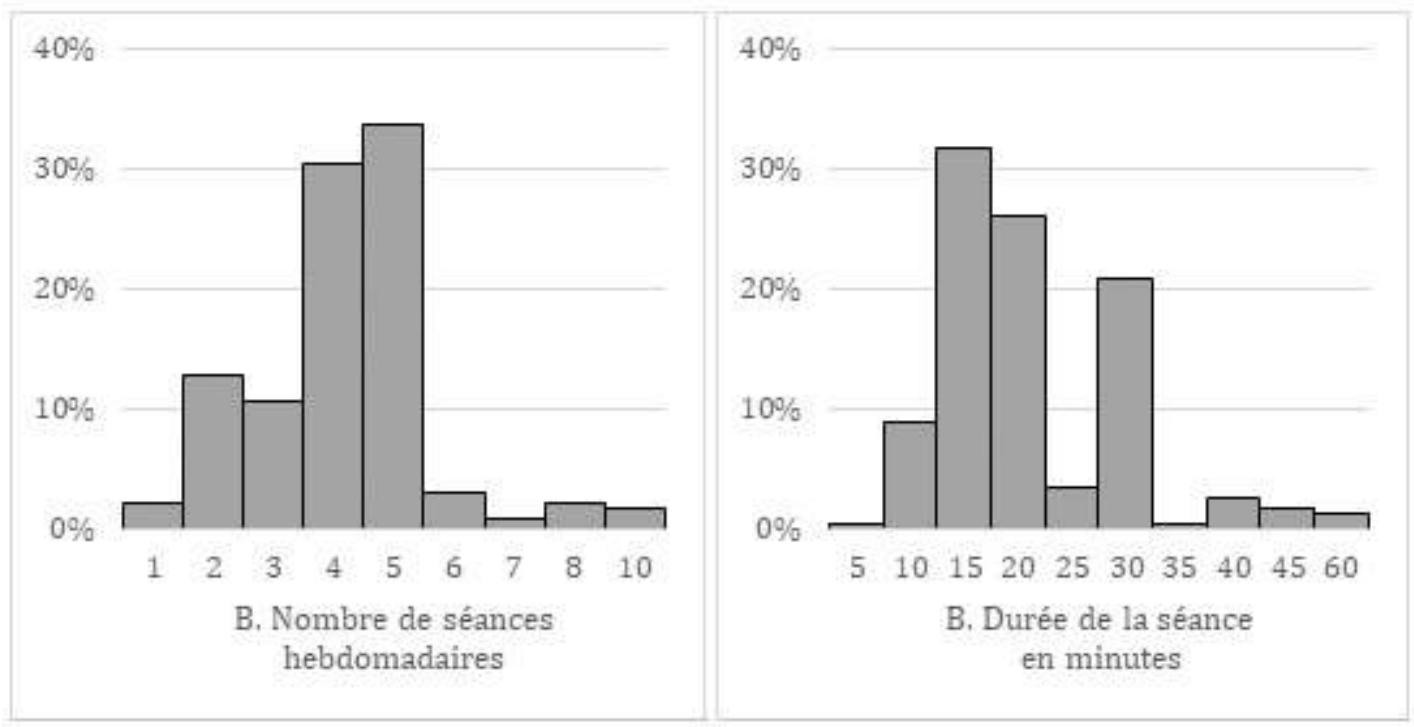


prévoient 4 ou 5 séances de 15, 20 ou 30 minutes (voir figure 1). Cette configuration se rapproche des préconisations des programmes avant 1977. Ainsi le volume horaire standard, qui ne représenterait cependant qu'environ la moitié des enseignants, serait de $1 \mathrm{~h}$ à $2 \mathrm{~h} 30$ par semaine.

\section{Les « stratégies » d'enseignement}

Les réponses données par les enseignants aux différentes questions liées à leur pédagogie seront analysées selon la dimension à laquelle elles font référence, forme, trajectoire et pression. Le tableau ci-dessous recense les pratiques des enseignants pour l'apprentissage de l'écriture. La question posée était la suivante: «Dans l'apprentissage de l'écriture cursive, avez-vous des stratégies particulières afin de faire comprendre à l'enfant la "façon de faire" ? » Chaque enseignant était libre de citer autant de méthodes ou moyens qu'il le souhaitait. Ceux-ci ont été regroupés selon qu'ils étaient basés sur le visuel, le verbal ou le toucher, et sont donnés en pourcentage d'enseignants ayant évoqué une stratégie donnée (soit 201 enseignants) ${ }^{3}$.

Une grande partie des enseignants insiste sur la nécessité « de ressentir le mouvement avec son corps " en traçant des lettres de grande taille en l'air. De nombreux actes pédagogiques de l'enseignant sont centrés directement sur le mouvement de l'enfant, qui se résume dans cette expression « nous travaillons l'enchaînement des gestes et non les lettres » plus fréquente chez les enseignants de GS. La réduction du mouvement intervient ensuite en passant par une variété de supports, le tableau, l'ardoise, les grandes feuilles, puis, essentiellement en $\mathrm{CP}$, une adaptation progressive aux lignages. Les stratégies gestuelles mettent clairement en évidence la progression d'un tracé qui doit être de plus en plus précis.

Parmi les stratégies impliquant une visualisation du tracé, quelque soit le support, ce sont des éléments de la trajectoire qui sont les plus utilisés. En effet, le sens du tracé, le point de départ sont les

Tableau 2. Types de stratégie rapportés par les enseignants pour expliciter les propriétés du tracé d'une lettre

\begin{tabular}{|c|c|c|}
\hline \multicolumn{2}{|c|}{ Stratégies d'enseignement } & \multirow{2}{*}{$\begin{array}{l}\begin{array}{l}\text { Pourcentage } \\
\text { d'enseignants }\end{array} \\
64 \%\end{array}$} \\
\hline Gestes & Gestes de l'enseignant/démonstration en l'air & \\
\hline \multirow{7}{*}{$\begin{array}{l}\text { Utilisation de modèles et } \\
\text { indications symboliques }\end{array}$} & Flèches & $42 \%$ \\
\hline & Point de départ & $18 \%$ \\
\hline & Chiffres & $7 \%$ \\
\hline & Graphisme/formes & $6 \%$ \\
\hline & Couleurs & $5 \%$ \\
\hline & Démonstration & $5 \%$ \\
\hline & Autres & $10 \%$ \\
\hline Lignes & Lignes colorées & $11 \%$ \\
\hline \multirow{3}{*}{$\begin{array}{l}\text { Mobilisation corporelle et } \\
\text { haptique de l'élève }\end{array}$} & Corps & $3 \%$ \\
\hline & Lettres en relief, pâte à modeler ou rugueuses & $6 \%$ \\
\hline & Tracés dans matériaux meubles (sable semoule) & $1 \%$ \\
\hline \multirow[t]{5}{*}{ Méthodes verbales } & Phrases descriptives & $25 \%$ \\
\hline & Verbalisation & $18 \%$ \\
\hline & Vocabulaire spécifique & $9 \%$ \\
\hline & Métaphore/image & $8 \%$ \\
\hline & Devinette/comptine/histoire/jeu & $7 \%$ \\
\hline Posture & Ajustement de la posture de l'enfant & $4 \%$ \\
\hline Écrit & Faire écrire l'enfant & $14 \%$ \\
\hline
\end{tabular}


informations données les plus fréquentes. Le point d'arrivée ainsi que les lignes, matérialisation de l'orientation dans l'espace, apparaissent aussi mais moins fréquemment. L'utilisation de formes et de modèles est rare.

Les stratégies verbales, souvent associées aux stratégies gestuelles, semblent avoir pour but de renforcer ces dernières. Il peut aussi être demandé à l'enfant de verbaliser et décrire son geste, ainsi que celui de son enseignant. Souvent dans les réponses des enseignants la description du geste utilise un vocabulaire spécifique, langage descriptif métaphorique prenant parfois la forme d'histoires ou de comptines. Deux niveaux de langage métaphorique peuvent être mis en évidence.

La première forme renvoie à des métaphores qui ont acquis le statut de métalangage, c'est-à-dire de vocabulaire spécifique pour décrire les propriétés de lettres. Cela renvoie au vocabulaire type de lettres à boucles, à ponts, à cannes, à vagues, à jambages, etc. Il s'agit de termes métaphoriques et imagés que l'on retrouve dans les ouvrages pédagogiques à destination des enseignants (Baraud et al., 2016) et qui servent à décomposer le tracé des lettres en fonction de leur forme mais aussi des gestes associés.

La seconde renvoie à des métaphores qui peuvent s'avérer très imagées. Ces métaphores peuvent prendre une dimension narrative historiettes ou comptines qui expriment le sens du tracé, sa trajectoire, comme le montrent les deux exemples suivants: "Je tourne, je trace la canne et je m'envole» et « je monte tout droit jusqu'à la ligne du ciel, je tourne et je redescends jusqu'à la ligne de la terre, là je donne la main à la lettre suivante ». On retrouve le langage imagé pour l'orientation de l'écrit dans les consignes associées au lignage : «suivre le chemin de terre, ne pas dépasser la hauteur de l'herbe pour les minuscules et le ciel pour les lettres hautes $»$.

De manière plus rare, toute une histoire peut être construite pour expliciter l'écriture d'un mot. Voici l'histoire racontée pour l'écriture du mot marou :

Je dis aux élèves :

Petite canne de grand père, je remonte sur le même trait, petit pont, je remonte sur le même trait et encore un petit pont avec une petite main : $\mathrm{m}$

Je commence au milieu du chemin, je tourne comme pour faire un rond, je m'accroche à la main du $\mathrm{m}$, je ferme et petite canne de parapluie : a

Je m'accroche à la main du a, je monte et je fais dépasser le bout du nez du r, à droite puis canne de parapluie : $\mathrm{r}$ Je commence en haut du chemin, je tourne comme pour un rond, je ferme et je rentre à l'intérieur et je fais la main du o en haut : o

Je m'accroche à la main du o puis je fais une canne de parapluie, je remonte en haut du chemin et je rafais une canne de parapluie : $\mathrm{u}$

Pour résumer, on constate donc que les stratégies majoritairement utilisées, qu'elles soient gestuelles, visuelles ou verbales sont centrées prioritairement sur le geste, sur sa dynamique, sur le processus menant à la formation de la lettre et peu sur ses caractéristiques morphologiques. Les enseignants apprennent aux enfants à réaliser un geste, non à reproduire un dessin. Leurs stratégies sont donc orientées dans ce sens. Plusieurs enseignants de GS comme de CP ont insisté sur l'importance d'une progression partant du corps tout entier : « engager tout le corps pour mémoriser les formes et le sens des lettres », ou encore « travailler l'orientation spatiale par des jeux avec le corps, puis les bras, puis la main ». Cet engagement du corps dans l'espace est progressivement canalisé par l'amplitude du mouvement qui va diminuer, passant de l'espace, au tableau, aux grandes feuilles (A3), à l'ardoise et à la feuille, feuille blanche tout d'abord, puis présence de lignes dont l'espacement va aller en diminuant jusqu'à la fin du CP. Cette incorporation de l'espace est renforcée par des indications verbales qui décomposent le geste en différentes étapes et les supports visuels qui explicitent sous forme de signes, flèches, pointillés, points, etc. Pour compléter ces stratégies, quelques rares enseignants utilisent également des outils sensoriels (bac de semoule, lettres rugueuses, pâte à modeler).

Une dernière méthode utilisée, le modèle. Bien que spontanément le rôle du modèle n'ait pas été évoqué explicitement dans la question ouverte sur des méthodes utilisées, lorsque l'on pose la question de comment travailler à partir d'un modèle, qui pour $80 \%$ des enseignants est un modèle écrit (à la main), trois types d'exercices, éventuellement consécutifs (pour les $2 / 3$ des enseignants) se combinent : on fait le geste en l'air (88\%), on repasse sur le modèle (59\%) et on le recopie ( $77 \%$ ).

Ces stratégies insistent sur les dimensions de la forme et de la trajectoire, la pression n'est pas mentionnée de manière explicite même si les exercices proposés font travailler ces trois dimensions. 


\section{L'apprentissage des lettres et de leur enchainement}

La dynamique du tracé d'une lettre ou d'une succession de lettres dépend malgré tout de la forme à tracer, ce que les enseignants expriment souvent clairement dans leur organisation de l'ordre d'apprentissage des lettres, ainsi que dans les difficultés des enchainements de lettres.

Si l'enseignement de l'écriture cursive est imposé par les programmes ministériels, seules les minuscules cursives font l'objet de ce que l'enfant doit maitriser en $\mathrm{CP}$, et moins de la moitié des enseignants (42\%) considèrent que les majuscules cursives doivent être acquises en CP, elles ne sont quasiment pas abordées en GS (8\% seulement des enseignants). Le type d'écriture enseigné diffère ainsi d'un niveau à l'autre. En GS et pour la quasi-totalité des enseignants, lettres capitales ou script et cursives minuscules font partie des apprentissages. Il est par ailleurs courant, tant en GS qu'en CP, d'associer dans un même mot majuscule scripte et minuscules cursives : la moitié des enseignants ont déclaré avoir recours à cette pratique.

Y a-t-il un ordre privilégié pour l'acquisition de la maitrise ? Si la grande majorité des enseignants (87\% d'entre eux) sont d'accord pour dire que l'apprentissage des lettres se fait dans un ordre particulier, il n'y a pas de consensus. Les critères avancés sont multiples, mais la plupart du temps justifiés. Il est intéressant cependant de noter que l'on retrouve une cohérence d'arguments selon le niveau, GS ou $\mathrm{CP}$ : c'est la forme et/ou du geste lié au tracé qui est déterminant en GS alors qu'au CP, l'ordre est plus souvent lié aux sons et à la progression de la lecture.

L'ordre alphabétique n'est quasiment jamais utilisé. Plusieurs types de regroupement sont avancés par les enseignants. Les regroupements liés au geste et à la forme des lettres sont les plus fréquents, basés soit sur un critère de similitude lié à des catégories de forme telles que les boucles, les ponts, les vagues, etc., soit sur un critère de complexité du geste. Un quart des enseignants, essentiellement en $\mathrm{CP}$, opère des regroupements liés aux sons associés aux lettres, souvent en suivant l'ordre de la méthode de lecture qu'ils utilisent mais aussi parfois en commençant par les voyelles et en poursuivant avec les consonnes. Un dixième d'entre eux utilise d'autres critères comme la fréquence des lettres dans la langue, en commençant par les plus utilisées, alors que d'autres se basent sur l'ordre fourni par des ouvrages et que les derniers, bien qu'ils ne suivent pas une logique explicite, semblent se baser sur une complexité croissante du graphisme de la lettre.

Certaines lettres présentent en effet une difficulté d'apprentissage plus importante pour les élèves. Ainsi, le f est la lettre qui revient le plus souvent (64\% des enseignants), suivi du k (59\%), du z (50\%). Les lettres b, h, y, v, w et $\mathrm{x}$ font quant à elles partie des plus difficiles pour plus de $20 \%$ des enseignants. La difficulté de maitrise de ces formes viendrait de la combinaison de boucles grandes et petites nécessitant un mouvement de rotation de la main ; elles sont bien souvent travaillées en fin d'apprentissage. À l'opposé, le c n'a jamais été proposé comme lettre difficile.

Ces difficultés liées à la rotation se retrouvent dans les enchainements. Parmi les 69 couples de lettres difficiles à tracer mentionnés, les sept premiers couples cités commencent par b ou v. Par ailleurs les successions « br » et « vr » arrivent très largement en tête des difficultés (cités respectivement par $54 \%$ et $40 \%$ des enseignants). La lettre $r$ n'étant pas considéré en soi comme difficile, c'est donc bien l'enchainement qui pose problème. On trouve ensuite « ve », « be », « vi », « bi », suivi de « vu », « bu ». Les couples commençant par la lettre o apparaissent en troisième position des lettres difficiles à enchainer, comme le sont mais dans une moindre mesure les s, $\mathrm{x}, \mathrm{w}$, et a.

Retrouve-t-on ces configurations d'enchainement lorsque l'on demande de citer des mots difficiles? Tout en offrant une grande diversité (121 mots différents ont été donnés) les réponses reflètent ces difficultés, les quatre mots les plus souvent cités contiennent justement l'enchainement «br », l'enchainement «vr » étant cité nettement moins fréquemment. On trouve ainsi les mois de l'année (septembre, octobre, novembre et décembre), ou encore bruit, bras, arbre, ou zèbre. Ces deux derniers mots sont particulièrement difficiles puisque qu'il faut enchainer r-b-r d'une part et «zèbre » implique en plus du couple «br», la lettre $z$ qui apparait en troisième position des lettres difficiles à apprendre. Les mots ayant une syllabe en « vr » comme livre, vrai ou vraiment, sont nettement moins fréquents. De manière surprenante la longueur des mots n'a été donnée que rarement comme source de difficulté pour les élèves, alors que sur les 121 mots différents proposés, un tiers comportent 6 lettres ou plus. 
En conclusion de cette section, arrêtons-nous sur une des questions posées concernant les éléments sur lesquelles il est nécessaire d'orienter l'attention de l'enfant. Dix éléments étaient proposés : fluidité, rapidité, lisibilité, tenue du crayon, posture, épaisseur du trait, organisation de l'espace de la feuille, forme et taille des lettres, sens de l'écriture. Le sens de l'écriture et la tenue du crayon font la quasiunanimité. On trouve ensuite la taille des lettres. Puis viennent la précision, la fluidité et l'organisation de la feuille. La régularité arrive loin derrière, tandis que la rapidité, l'esthétique et l'épaisseur du trait ne sont pratiquement jamais citées. On constate donc que l'on retrouve bien ici l'une des composantes de l'écriture essentielles dans les stratégies d'enseignement, le sens de l'écriture qui donne la trajectoire de la trace. La tenue du crayon de même que la posture peuvent être interprétées comme des conditions nécessaires à la maitrise non seulement de la forme et taille des lettres, mais sans doute aussi de la pression du crayon sur le support.

\section{Supports matériels privilégiés}

L'ensemble des éléments matériels à disposition de l'enfant sont autant d'éléments qui vont structurer son espace d'actions encouragées. Les enseignants ont à disposition une panoplie d'outils allant des outils « traditionnels »- tableau noir ou blanc, ardoise (respectivement par $61 \%, 55 \%, 85 \%$ ), des cahiers d'exercices (59\%) - aux moyens numériques qui restent peu disponibles. En effet, seuls $16 \%$ des enseignants disposent d'un tableau interactif, moins de $10 \%$ de vidéo ou de vidéoprojecteur. Enfin, $45 \%$ des enseignants réalisent eux-mêmes d'autres moyens plus diversifiés, tels que des fiches ou des supports plastifiés permettant d'écrire au feutre.

Quant à l'utilisation des différents outils à disposition, l'ardoise reste plébiscitée. Dans une succession de questions sur l'importance (sur une échelle allant de 1 à 10, 1 étant le plus important) attribuée aux supports dans les exercices d'écriture, $79 \%$ considèrent l'ardoise comme très importante (évaluation 1 et 2 sur l'échelle de 10), de même que l'écriture au tableau qu'il soit noir ( $50 \%$ ) ou blanc (62\%). Les cahiers d'exercices et les feuilles lignées sont considérés comme importantes ( $50 \%$ et $58 \%$ respectivement), les feuilles blanches et à carreaux n'étant vues comme importantes que par environ $30 \%$ des ensei- gnants. En revanche tablette, ordinateur ou même TNI n'apparaissent pas comme des outils favorables à l'apprentissage de l'écriture. L'outil scripteur bien entendu va de pair avec le support. Le feutre et la craie sont associés au tableau et à l'ardoise. Pour les supports papier, le crayon papier est préféré par deux tiers des enseignants, alors que le stylo bille est peu utilisé (9\%) et que le stylo plume a quasiment disparu du matériel de l'élève scripteur débutant !

Le support et la taille ne sont pas indépendants, ils évoluent conjointement au cours de l'apprentissage. L'ardoise et le tableau permettent d'écrire gros, alors que les cahiers et les supports élaborés par les enseignants ont des tailles progressives. Ainsi, $78 \%$ des enseignants évoquent la diminution progressive de la taille des interlignes des supports présentés aux élèves, pour atteindre à la fin de l'année de $\mathrm{CP}$ ou en CEl une taille cible de $2 \mathrm{~mm}$ qui correspond à l'interligne des réglures Seyès. Par exemple, une enseignante de CP décrit les étapes suivantes : "Lettre géante sur un tableau imaginaire avec le doigt, lettre moyenne sur ardoise blanche et crayon effaçable, lettre dans interligne sur ardoise. " La diminution progressive de la taille des lettres était déjà présente dans les programmes de 1882 où les enfants apprenaient à écrire en gros, en moyen et en fin au cours élémentaire. Une analyse des cahiers à destination des élèves édités par plusieurs éditeurs français a montré la présence de cette diminution progressive de la taille des interlignes, sans qu'il y ait de norme particulière (Baraud et al., 2016).

\section{Cas particulier des cahiers Seyès}

La réglure Seyès, créée en 1892, est une spécificité française et consiste en des lignes horizontales espacées de $2 \mathrm{~mm}$, et des lignes verticales tous les $8 \mathrm{~mm}$, alors que dans la plupart des pays si un lignage double ou triple est utilisé au moment de l'apprentissage, celui-ci fait rapidement place à un lignage simple. Nous avons voulu savoir de quelle manière les enseignants l'utilisaient, nous leur avons donc demandé quelle était son utilité et comment les élèves apprenaient à écrire avec ce support. Cette réglure conditionne la gestion de l'espace de plusieurs manières. Les lignes et interlignes horizontaux sont utilisés par les enseignants pour travailler la hauteur des lettres. Ainsi par exemple le t et le d ont une hauteur de deux interlignes, le l et le b ont 
une hauteur de trois interlignes, le a, le o, le n ont une hauteur d'un interligne, etc. Une grande partie des enseignants (66\%) s'accorde sur ce point. Elles sont également utilisées par $21 \%$ des enseignants pour amener les élèves à maintenir l'horizontalité de l'écriture. En revanche, le rôle des lignes verticales est moins clair. Pour $39 \%$ des répondants, elles servent de guide, lié à la présentation et ils s'appuient souvent sur la marge pour débuter les phrases ; $15 \%$ d'entre eux les utilisent pour aider l'élève à maintenir la verticalité des lettres à boucle ou à jambage haut ou bas ${ }^{4}$ et pour $12 \%$ d'entre eux, les carreaux sont utilisés comme des repères d'espacement. La réglure, et en particulier la réglure Seyès est donc utilisée dans les exercices afin d'apprendre à l'élève les proportions de l'écriture et le repérage dans la page. Toutefois, certains enseignants ont déclaré ne pas percevoir l'utilité de la ligne verticale.

\section{Évaluation et difficultés}

Parmi les 10 composantes caractéristiques de l'activité d'écriture (fluidité, rapidité, lisibilité, tenue du crayon, posture, épaisseur du trait, organisation de l'espace de la feuille, forme et taille des lettres, sens de l'écriture) il était demandé d'évaluer le degré de difficulté. Pour $90 \%$ des enseignants la tenue du crayon et le sens du tracé apparaissent comme les dimensions essentielles sur lesquelles l'enfant doit porter son attention, viennent ensuite la taille des lettres et la lisibilité ( $74 \%$ et $54 \%$ des enseignants respectivement). La fluidité, la régularité ou l'organisation de l'espace sont mentionnés par environ un tiers des réponses, l'esthétique ou la rapidité ne sont pas prioritaires.

Pour prévenir ou remédier à ces difficultés, l'analyse des réponses libres a permis de dégager différents types de stratégies dont l'objectif vise d'une part à corriger ou anticiper les erreurs et d'autre part à tenter une amélioration des habiletés de l'enfant. Dans leurs réponses les enseignants insistent sur l'importance de l'entrainement et de la répétition (6\%) et la mise en place d'un accompagnement personnalisé des élèves (14\%) en consacrant une partie des leçons à des travaux individuels ou en petit groupe. La tenue du crayon et la posture apparaissent comme critiques par le quart des enseignant qui considèrent qu'il est nécessaire d'être vigilant dès la petite section sur la position du corps et la tenue du crayon. Nombreuses sont les propositions d'utilisation de « guide doigt », de crayons triangulaires ou encore d'embouts ergonomiques, une mauvaise tenue du crayon pouvant avoir une répercussion sur le sens du tracé, l'organisation de l'espace et la fluidité. Ils peuvent pour cela s'appuyer sur différents outils comme des lignages multicolores (un enseignant sur dix) pour travailler la gestion de l'espace. Ces différents outils peuvent être associés à la verbalisation du geste et au renforcement des modèles fléchés pour insister sur la trajectoire. Une recommandation se fait jour, laisser le temps : travailler par étape, aller doucement, ne pas commencer trop tôt l'écriture cursive, « attendre que les doigts soient habiles".

$\mathrm{Au}$ vu de ces réponses, les outils utilisés ne diffèrent pas de ceux cités pour l'enseignement en général, les enseignants semblent plutôt avoir recours à un renforcement de leurs méthodes habituelles lorsqu'un élève est en difficulté. Il semblerait donc que les difficultés rencontrées couramment par les élèves soient prises en charge non pas à travers des dispositifs spécifiques mais par un accroissement du temps d'accompagnement et du temps d'entrainement.

\section{Cursive, outils numériques et avenir de l'écriture}

En 2011 paraissait dans la revue scientifique Journal of Motor Behavior, un article au titre évocateur « La mort de l'écriture manuscrite : effets secondaires sur la motricité de l'utilisation fréquente de l'ordinateur » (Salzenbruck, Hegele, Rinkenauer et Heuer, 2011) $)^{5}$. Les auteurs concluaient ironiquement sur « une espèce en danger : les humains maitrisant l'écriture »! Des articles de presse, nombreux et plus ou moins polémiques laissent entendre que la Finlande abandonnerait l'écriture manuscrite au profit du clavier ${ }^{6}$, ce qui est par ailleurs tout à fait inexact puisque l'apprentissage de l'écriture cursive y serait facultatif alors que l'écriture script reste une discipline enseignée pour tous.

Que disent les programmes français? L'utilisation des outils numériques apparait dans les programmes dans les années 1980 à travers l'initiation au traitement de texte, les programmes de 1995 mentionnent " l'ordinateur au service des disciplines », celuici étant cité comme support au même titre que les dictionnaires, ou les jeux éducatifs. Avec le 
programme de 2008, les élèves dès le CP sont « amenés à utiliser l'ordinateur : écriture au clavier, utilisation d'un dictionnaire électronique ». C'est dans les programmes publiés en novembre 2015 que l'utilisation du clavier est mentionnée comme complémentaire ou même interchangeable avec l'écriture cursive, une fois l'écriture manuscrite acquise :

Ayant commencé d'apprendre à écrire (en cursive, au clavier) en GS, ils complètent l'apprentissage du geste graphomoteur non achevé et perfectionnent leurs acquis (sureté et vitesse), automatisant progressivement le tracé normé des lettres. Ils apprennent à utiliser les fonctions simples d'un traitement de texte, ils manipulent le clavier. De façon manuscrite ou numérique, ils apprennent à copier ou transcrire sans erreur, depuis des supports variés (livre, tableau, affiche...) en veillant à la mise en page. (...) Le recours aux outils numériques (traitement de texte avec correcteur orthographique, dispositif d'écriture collaborative...) peut permettre d'alléger la tâche de rédaction et de relecture.

Il est donc apparu que le point de vue des enseignants concernant l'avenir de l'écriture cursive était un élément potentiellement important pour mieux comprendre leur attitude et leurs pratiques du numérique.

\section{Les outils numériques}

Une grande majorité d'enseignants (63\%) répondent par l'affirmative lorsqu'on leur demande si les outils numériques peuvent avoir un impact sur l'apprentissage de l'écriture cursive et du geste graphomoteur. Cette question ne laisse manifestement pas indifférent, et les avis divergent. Alors que la question était centrée sur le rôle des outils numériques pour l'apprentissage de l'écriture cursive, elle a aussi renvoyé à l'idée de l'utilisation du clavier comme substitut à l'écriture manuscrite cursive. Si de nombreux enseignants y voient une gêne à l'apprentissage de l'écriture cursive, d'autres vont dans le sens d'un transfert de l'écriture manuscrite cursive comme moyen de communication vers le numérique, ce qui peut pallier les difficultés de certains enfants, en particulier dans le cas de dyspraxie. En outre la possibilité de garder une trace de l'écrit est perçue positivement, conduisant à une visualisation du geste
« juste» qui permettrait d'afficher plus explicitement les corrections à apporter aux tracés. D'autres aspects favorables à l'utilisation d'outils numérique sont parfois invoqués : leur composante ludique serait motivant pour l'enfant, et l'on trouve aussi l'argument de la quasi disparition, dans la vie quotidienne, de textes manuscrits, la plus grande partie de la correspondance étant saisie sur clavier.

Afin d'approfondir la question, nous avons interrogé les enseignants à propos de l'abandon de l'écriture cursive en Finlande et, bien que seule une petite moitié ( $46 \%$ ) ait connaissance de cette décision, la plupart des enseignants expriment un fort désaccord avec cette évolution de l'enseignement de l'écriture. Les raisons sont souvent liées à la crainte d'un impact négatif sur le développement de la motricité fine des élèves, de la coordination manuelle, mais aussi des capacités d'attention, de mémorisation, d'orthographe ou encore d'effort. Le geste graphique est de même souvent associé à une facilitation de l'apprentissage de la lecture, voire pour quelquesuns, au développement du cerveau. L'intérêt de l'écriture cursive est également associé à l'apprentissage et à la vie quotidienne, notamment du fait de sa rapidité. En effet les enseignants semblent distinguer son utilité pratique quotidienne qualifiée par les adjectifs " nécessaire ", "indispensable », mais aussi « rapide », " adaptable », de son utilité comme médiatrice de l'esthétique, du beau, ou même du plaisir.

Une enseignante évoque également l'importance du " griffonnage » qui souvent accompagne la réflexion nécessaire à la production écrite : «Bien souvent l'inspiration vient avec le geste d'écriture [...]. Cela aide à clarifier ses idées lorsqu'on peut griffonner, barrer, etc. ». Pour d'autres, la capacité à prendre en note rapidement une information sera toujours nécessaire.

Quelques enseignants cependant seraient plutôt d'accord car ils perçoivent l'abandon de la cursive au profit du clavier comme une adaptation aux exigences sociales actuelles, permettant aux enfants d'acquérir une compétence indispensable aujourd'hui. Pour d'autres l'écriture cursive est difficile à apprendre et son utilité peut être mise remise en question. Ainsi, à l'heure des outils numériques, l'apprentissage de l'écriture cursive est une source de débat et d'interrogations pour les enseignants en charge de son enseignement. 


\section{DISCUSSION}

Dans le but d'évaluer la manière dont les enseignants déclarent construire "l'espace d'actions» de l'enfant qui apprend à écrire, une enquête par internet sous forme de questionnaire a été proposée à l'ensemble des classes de grande section (GS) et de cours préparatoire (CP) des écoles maternelles et primaires de deux académies de la région Rhône-Alpes-Auvergne, résultant en 226 réponses utilisables. L'objectif était de mettre en relation la manière dont les enseignants évaluent des difficultés de l'écriture cursive avec leurs pratiques de cet enseignement, et de voir dans quelle mesure ces pratiques facilitent l'appropriation par l'enfant des composantes fonctionnelles de l'écriture - dynamique de la trajectoire (sens et direction) et pression de l'outil scripteur sur le support. Un ensemble de questions, dont un certain nombre étaient présentées sous forme de questions ouvertes, a permis d'évaluer le temps dédié à cet enseignement, les ressources matérielles relatives à l'écriture dont disposent les classes, les stratégies d'enseignement des enseignants, ainsi que leur évaluation des difficultés dans le tracé des lettres et des mots. D'autres questions avaient pour but d'explorer la manière dont les enseignants interprètent les recommandations des instructions officielles concernant l'apprentissage de l'écriture en GS et $\mathrm{CP}$ dans leur pratique enseignante, de même que leur recours à ressources autres tels que les manuels ou livres du maitre.

Bien qu'ils déclarent ne pas être attachés à des méthodes particulières, les enseignants interrogés font état de pratiques qui apparaissent tout à la fois diversifiées, et paradoxalement relativement proches. Le recours à une variété de ressources leur permet de combiner un ensemble d'approches méthodologiques diverses en réalisant une sorte de « bricolage » pédagogique aboutissant à des méthodes plus ou moins personnalisées, adaptables aux spécificités de chaque élève et aux différents moments de l'apprentissage, ce qu'exprime assez bien une enseignante :

Pas de méthode académique mais par contre je m'en suis inventée une...

L'apprentissage est hyper personnalisé : je différencie grâce au tableau noir ou blanc selon ce qu'il y a dans la classe. Un enfant qui arrive à faire son travail sur son ardoise peut passer au travail papier. Pour celui pour qui c'est plus dur, il vient au tableau avec moi : il peut repasser un modèle que j'ai fait en grand puis s'il s'en sent capable, refaire la lettre à côté le plus de fois possible afin que le geste devienne automatique.

Globalement, les enseignants organisent un apprentissage de difficulté progressive qu'il s'agisse de la complexité du tracé ou de la taille imposée des lettres. Par ailleurs, nous avons pu voir que la majorité d'entre eux disent prêter attention à la taille et au sens de l'écriture, ce qui rejoint les résultats de l'enquête réalisée par de Baraetal (2011) auprès d'enseignants québécois et français.

En outre, deux caractéristiques de l'enseignement se font jour : diversification des supports - tableau, ardoise, lignages variés - d'une part et d'autre part des stratégies d'enseignement permettant l'appropriation de la dynamique de la forme basées sur le geste et/ou la verbalisation avec, notamment, un recours important à un métalangage de l'écriture fait de métaphores. Nous retrouvons donc ici des principes pédagogiques en adéquation avec les principes de Bernstein pour qui un haut niveau de maitrise correspond à une capacité d'adaptation de la réalisation de l'action à des conditions variés d'environnement, l'apprentissage consistant en ce qu'il décrivait comme des « répétitions sans répétition » (Bernstein, 1996 ; Biryukova et Bril, 2002). Pour cet auteur, l'apprenant doit expérimenter une grande diversité de situations ce qui en retour va conduire à une grande diversité de sensations qui vont permettre une meilleure adaptation à un environnement changeant. On peut donc penser que les stratégies d'enseignement très variées décrites par les enseignants créent des conditions favorables au développement de cette capacité d'adaptation.

Si l'on considère à présent les composantes fonctionnelles retenues pour décrire la fonctionnalité de l'action graphomotrice, on constate que les stratégies des enseignants sont focalisées essentiellement sur le contrôle de la trajectoire. Le sens de l'écriture semble aller de soi, du moins pour les droitiers, mais nous n'avons pas abordé dans le questionnaire les particularités de l'apprentissage par les enfants gauchers. En revanche la composante de pression, pourtant fondamentale puisque c'est celle qui est à l'origine de la trace proprement dite, est rarement mentionnée. Elle est apparue avec des remarques sur l'utilisation du stylet sur les tablettes, présentées comme des surfaces glissantes n'ayant que peu de points communs avec l'écriture «papier-crayon », qui reste le prototype du 
support de l'écriture manuscrite cursive. Toutefois, bien qu'ils ne fassent pas référence explicitement aux différentes recherches menées sur les processus en jeu dans l'apprentissage de l'écriture, les enseignants vont dans le sens de ces travaux pour le choix de leurs méthodes. En particulier, il ressort des résultats de l'enquête qu'un entrainement multisensoriel et notamment haptique est largement plébiscité ; or selon Bara et Gentaz (2011), ce type d'entrainement serait particulièrement efficace pour l'acquisition du sens de l'écriture. Ainsi, bien que les enseignants ne mentionnent pas explicitement les deux composantes direction et pression, celles-ci sont travaillées à travers les exercices mobilisant et combinant plusieurs modalités sensorielles qu'ils ont déclaré utiliser avec leurs élèves.

Enfin, les questions relatives à l'introduction des outils numériques dans la cadre de l'apprentissage de l'écriture cursive et à l'abandon de son apprentissage en Finlande ont été tout particulièrement intéressantes car elles ont permis aux enseignants d'exprimer les raisons de leur attachement à l'apprentissage de l'écriture manuscrite cursive ainsi de leurs craintes quant à l'utilisation du numérique et de tablettes en particulier. La grande majorité des enseignants voit de manière très négative l'abandon de l'écriture cursive en Finlande, bien que certains relativisent cet abandon auquel est substitué, comme cela est le cas aux États-Unis, l'écriture script.

\section{CONCLUSION}

En conclusion il nous semble nécessaire de soulever la question de l'interface entre recherche et enseignement. À la suite de ce travail il apparait assez clairement, et en cela les résultats de notre enquête rejoignent les conclusions de notre analyse des manuels et livres du maitre (Bril et al., 2016), ainsi que des instructions officielles (Baraud et al., 2016), ou encore celles de Labrecque, Morin et MontésinosGelet (2013), qu'il est très peu fait référence aux travaux scientifiques pour légitimer ou questionner les pratiques d'enseignement par les acteurs euxmêmes. Certes la consultation d'un ensemble de chercheurs pour l'élaboration des programmes de 2015 semble indiquer une évolution positive des liens entre recherche et instructions officielles. Cependant, l'analyse des ressources utilisées montre que ce sont les sites internet et l'expérience personnelle qui sont le plus souvent invoquées comme aides à l'enseignement alors que la formation, la recherche, les livres et revues sont très peu cités et que les instructions ministérielles apparaissent souvent peu claires.

\section{NOTES}

1. La recherche présentée ici fait partie d'un projet plus large dont l'objectif est de proposer une étude des processus d'apprentissage en parallèle avec les pratiques de transmission mises en ouvre qui rendent possible et assistent ce processus. Réalisé dans le cadre de l'appel à projet ANR Apprentissage 2013, ce programme de recherche s'intitule « Le geste technique et son apprentissage " et a pour objectif d'évaluer les parentés des processus d'apprentissage et de transmission de gestes techniques complexes au-delà de la diversité de leurs formes et des contextes culturels dans lesquels ils se déroulent. Un des axes de recherche traités concerne l'acquisition du geste graphomoteur. Par ailleurs, les autrices ont fait le choix d'employer dans cet article la nouvelle orthographe issue de la réforme de 1990.

2. Pour une discussion sur la notion de contrainte fonctionnelle d'une tâche voir Bril et al., (2012) ou Bril (2015).

3. La question des stratégies sera également discutée partir d'une analyse des actes pédagogiques filmées durant la classe dans des publications ultérieures.

4. La verticalité est importante d'après ces réponses, ce qui nous amène à nous interroger sur l'adéquation entre la police proposée par le ministère, qui est une police penchée à caractère plutôt nordique (MEN, 2013) et les modèles et pratiques effectifs des enseignants de classes d'écoles primaires.

5. Sulzenbruck et al. (2011), The Death of Handwriting : Secondary Effects of Frequent Computer Use on Basic Motor Skills, Journal of Motor Behaviour, 43, p. 247-251.

6. http://www.bbc.com/news/blogs-news-from-elsewhere-30146160

\section{RÉFÉRENCES}

Asher, A. V. (2006). Handwriting instruction in elementary schools. American Journal of Occupational Therapy, 60, p. 461-471.

Bara, F., et Gentaz, E. (2010). Apprendre à tracer les lettres : une revue critique. Psychologie Française, 55, p. 129-144. 
Bara, F., et Gentaz, E. (2011). Haptics in teaching handwriting: The role of perceptual and visuo-motor skills. Human Movement Science, 30, p. 745-759.

Bara, F., Morin, M.-F., Montésinos-Gelet, I., \& Lavoie, N. (2011). Conceptions et pratiques en graphomotricité chez des enseignants de primaire en France et au Québec. Revue française de pédagogie, p. 41-56.

Baraud, M., Bril, B., Acioly-Régnier, N., Hoibian, S. (2016). L'apprentissage de l'écriture: des instructions officielles aux outils pour la classe en CP et GS. Communication présentée au colloque international « Enseignement et apprentissage de l'écriture de la maternelle à l'université et dans les formations tout au long de la vie » à ESPE d'Aquitaine, université de Bordeaux, France.

Bernstein, N. (1967). The Coordination and Regulation of Movements. London : Pergamon Press.

Bernstein, N. A. (1996). Dexterity and Its Development. Dans M. Latash (dir.), Dexterity and its development (p. 1-235). Marwah, NJ : Lawrence Erlbaum Associates.

Biryukova, E. V., Bril, B. (2002). Bernstein et le geste technique. Dans B. Bril et V. Rroux (dir.), Le geste technique. Réflexions méthodologiques et anthropologiques (p. 49-68). Ramonville Saint-Agne : Éditions Erès.

Bosga-Stork, I. M., Bosga, J., et Meulenbroek, R. G. J. (2011). Intentional control and biomechanical exploitation in preparatory handwriting. Human Movement Science, 30, p. 687-697.

Bril, B. (2015). Learning to use tools: a functional approach to action. Dans L. Filletaz \& S. Billet (dir.), Learning through and for practice: contributions from Francophone perspectives (p. 95-118). Springer.

Bril, B., Smaers J., Steele, J., Rein, R., Nonaka, T., Dietrich, G., Biryukova, E., Hirata, S., Roux, V. (2012). Functional mastery of percussive technology in nut cracking and stone flaking: experimental data and implication for the human brain. Philosophical Transaction of the Royal Society: Biology, 367(1585), p. 59-74.

Chartrel, E., et Vinter, A. (2006). Rôle des informations visuelles dans la production de lettres cursives chez l'enfant et l'adulte. L'Année Psychologique, 106, p. 43.

Chartrel, E., et Vinter, A. (2008). The impact of spatiotemporal constraints on cursive letter handwriting in children. Learning and Instruction, 18, p. 537-547.

Dinehart, L. H. (2015). Handwriting in early childhood education: Current research and future implications. Journal of Early Childhood Literacy, 15, p. 97-118.

Feder, K. P., et Majnemer, A. (2007). Handwriting development, competency, and intervention. Developmental Medicine and Child Neurology, 49, p. 312-317.

Gibson, J. J. (1966). The senses considered as perceptual systems. Oxford, England: Houghton Mifflin.

Gimbert, F., Gentaz, É., et Mazens, K. (2013). Évaluation d'entrainements multisensoriels de préparation aux apprentissages numériques chez les enfants scolarisés en grande section de maternelle. ANAE. Approche neuropsychologique des apprentissages chez l'enfant, p. 189-196.
Graham, S., Harris, K. R., Mason, L., Fink-Chorzempa, B., Moran, S., et Saddler, B. (2007). How do primary grade teachers teach handwriting? A national survey. Reading and Writing, 21, p. 49-69.

Karlsdottir, R. (1996). Development of cursive handwriting. Perceptual and motor skills, 82, p. 659-673.

Longcamp, M., Zerbato-Poudou, M.-T., et Velay, J.-L. (2005). The influence of writing practice on letter recognition in preschool children: A comparison between handwriting and typing. Acta psychologica, 119, p. 67-79.

Lurçat, L. (1983). Le graphisme et l'écriture chez l'enfant. Revue française de pédagogie, 65, p. 7-18.

Maldarelli, J. E., Kahrs, B. A., Hunt, S. C., et Lockman, J. J. (2015). Development of early handwriting: Visualmotor control during letter copying. Developmental Psychology, 51, p. 879-888.

Meulenbroek, R. G. J., et Van Galen, G. P. (1988). The Acquisition of Skilled Handwriting: Discontinuous Trends in Kinematic Variables. Advances in Psychology, 55, p. 273-281.

Reed, E. S., et Bril, B. (1996). The Primacy of Action in Development. Dans M. L. Latash et M. T. Turvey (dir.), Dexterity and Its Development (p. 431-451). Mahwah, NJ: Lawrence Erlbaum Associates.

Reed, E. S. (1993). The intention to use a specific affordance: A conceptual framework for psychology. Development in context: Acting and thinking in specific environments, p. 45-76.

Ritchey, K. D. (2007). The building blocks of writing: Learning to write letters and spell words. Reading and Writing, 21, p. 27-47.

Sheffield, B. (1996). Handwriting: A neglected cornerstone of literacy. Annals of Dyslexia, 46, p. 21-35.

Sülzenbrück, S., Hegele, M., Rinkenauer, G., et Heuer, H. (2011). The Death of Handwriting: Secondary Effects of Frequent Computer Use on Basic Motor Skills. Journal of Motor Behavior, 43, p. 247-251.

Valsiner, J., et Van der Veer, R. (1993). The encoding of distance: The concept of the "zone of proximal development" and its interpretations. Dans R. R. Cocking et K. A. Renninger, The Development and Meaning of Psychological Distance (p. 35-62). Hillsdale, New Jersey, EUA: Lawrence Erlbaum Associates.

Velay, J.-L., Longcamp, M., et Zerbato-Poudou, M.-T. (2005). Apprendre à écrire les lettres pour mieux les reconnaître. Dans C. Thinus-Blanc \& J. Bullier (dir.), Agir dans l'espace. Paris : Éditions de la Maison des sciences de l'homme, p. 255-270.

Vinter, A., et Chartrel, E. (2010). Effects of different types of learning on handwriting movements in young children. Learning and Instruction, 20, p. 476-486.

Watkins, M., et Noble, G. (2011). Losing touch: pedagogies of incorporation and the ability to write. Social Semiotics, 21, p. 503-516. 